\title{
Design Guidelines for Improved Human-Robot Interaction
}

\author{
Jill L. Drury \\ The MITRE Corporation \\ Mail Stop K320 \\ 202 Burlington Road \\ Bedford, MA 01730 \\ jldrury@mitre.org
}

\author{
Dan Hestand and Holly A. Yanco \\ Computer Science Dept. \\ U. of Massachusetts Lowell \\ One University Avenue \\ Lowell, MA 01854 \\ \{phestand, holly\}@cs.uml.edu
}

\author{
Jean Scholtz \\ National Institute of Standards and \\ Technology \\ 100 Bureau Drive, MS 8940 \\ Gaithersburg, MD 20899 \\ jean.scholtz@nist.gov
}

\begin{abstract}
This poster highlights one of our analysis frameworks for human-robot interaction (HRI) and a sample of our results. We developed a fine-grained definition of HRI awareness based on research from computer-supported cooperative work (CSCW). Results consist of initial design guidelines for HRI that we are incorporating in next-generation robots and interfaces under development at UMass Lowell.
\end{abstract}

CATEGORIES AND SUBJECT DESCRIPTORS: H.5.2 [Information Interfaces and Presentation]: User Interfaces Screen Design; I.2.9 [Artificial Intelligence]: Robotics Operator Interfaces.

GENERAL TERMS: Design; Human Factors.

\section{INTRODUCTION}

Although much progress has been made in robotics in the last decade, relatively little progress has been made in optimizing the partnership between people and robots through improved techniques for HRI. Our work aims to fill this gap using techniques from $\mathrm{HCI}$ and $\mathrm{CSCW}$.

\section{HRI AWARENESS}

Much research has been performed in the CSCW community to characterize awareness; we adapted the concept [1] to account for all combinations of single and multiple humans and robots, as well as the non-symmetrical nature of the human-robot collaboration. Given $\mathrm{n}$ humans and $\mathrm{m}$ robots working together synchronously, we defined HRI awareness to have the following five components:

- Human-robot: the understanding that humans have of the locations, identities, activities, status and surroundings of the robots; and level of certainty regarding this data.

- Human-human: the understanding that humans have of the locations, identities and activities of their fellow human collaborators.

- Robot-human: the knowledge that the robots have of the humans' commands necessary to direct their activities and any human-delineated constraints that may require a modified course of action or command noncompliance.

- Robot-robot: the knowledge that the robots have of the commands given to them, if any, by other robots, the tactical plans of the other robots, and the robot-to-robot

Copyright is held by the author/owner(s).

CHI 2004, April 24-29, 2004, Vienna, Austria.

ACM 1-58113-703-6/04/0004. coordination necessary to dynamically reallocate tasks among robots if necessary.

- Humans' overall mission awareness: the humans' understanding of the overall goals of the joint human-robot activities and the moment-by-moment measurement of the progress obtained against the goals.

\section{PRELIMINARY DESIGN GUIDELINES}

We found that all anomalies could be traced to a lack of HRI awareness. Thus, when we developed a preliminary set of guidelines [3] for designing HRI in robots, we began with awareness and also included guidelines to address other major problems we observed with HRI:

- Enhance awareness. Provide a map of where the robot has been. Provide more spatial information about the robot in the environment to make operators more aware of their robots' immediate surroundings.

- Lower cognitive load. Provide fused sensor information to avoid making the user fuse the data mentally.

- Increase efficiency. Provide user interfaces that support multiple robots in a single window, if possible. In general, minimize the use of multiple windows.

- Provide help in choosing robot modality. Provide the operator assistance in determining the most appropriate level of robotic autonomy at any given time.

We have designed an interface prototype that incorporates the first three guidelines [2]. In particular, we have created a single display that overlays color video with heat indications, carbon dioxide measurements, and direction of the loudest local sound.

\section{ACKNOWLEDGMENTS}

The work was supported in part by NSF IIS-0308186, NIST 70NANB3H1116, and the DARPA MARS program.

\section{REFERENCES}

1. Drury, J., J. Scholtz and H.A. Yanco (2003). Awareness in Human-Robot Interactions. In Proceedings of the IEEE Conference on Systems, Man and Cybernetics.

2. Hestand, D. and H.A. Yanco. Layered Sensor Modalities for Improved Feature Detection. Submitted to ICRA-04.

3. Yanco, H.A., J.L. Drury and J. Scholtz. Beyond Usability Evaluation: Analysis of Human-Robot Interaction at a Major Robotics Competition. To appear in the Journal of Human-Computer Interaction in 2004. 